\title{
Activation of goat primordial follicles in vitro: Influence of alginate and ovarian tissue
}

\author{
Hudson H. V. Correia ${ }^{1}$ | Laritza F. Lima ${ }^{1}$ | Francisca Geovania C. Sousa ${ }^{1}$ | \\ Anna Clara A. Ferreira $^{1}$ | Jesus Cadenas ${ }^{1}$ | Victor M. Paes $^{1}$ | Benner G. Alves ${ }^{1}$ | \\ Ariella Shikanov $^{2}$ | José Ricardo Figueiredo ${ }^{1}$
}

\author{
${ }^{1}$ Laboratory of Manipulation of Oocytes \\ and Preantral Follicles (LAMOFOPA), State \\ University of Ceará, Fortaleza, Brazil \\ ${ }^{2}$ Department of Biomedical Engineering, \\ University of Michigan, Ann Arbor, MI, USA \\ Correspondence \\ José Ricardo Figueiredo, Laboratório \\ de Manipulação de Oócitos e Folículos \\ Pré-Antrais (LAMOFOPA), Universidade \\ Estadual do Ceará (UECE). Av. Silas \\ Munguba, 1700, Campus do Itaperi, CEP: \\ 60740903 Fortaleza - CE - Brasil. \\ Email: jrf.lamofopapapers@gmail.com \\ Funding information \\ Conselho Nacional de Desenvolvimento \\ Científico e Tecnológico, Grant/Award \\ Number: N 407594/2013-2
}

\begin{abstract}
The present study aimed to evaluate the effect of three culture systems on caprine primordial follicle activation in vitro: follicles cultured either in the isolated form within alginate (Isolated follicles + Alginate treatment), or enclosed in ovarian tissue (in situ), with or without alginate (Fragment + Alginate, and Fragment alone treatments, respectively). After culture, the Isolated follicles + Alginate treatment presented a percentage of morphologically normal follicles (MNF) similar to both the non-cultured control and the Fragment Alone treatments. Nevertheless, Fragment + Alginate treatment showed a significant reduction in the number of MNF when compared to the other treatments. Regarding follicle development, our results showed that regardless of the alginate, the presence of ovarian tissue limited primordial follicle activation during in vitro culture. Remarkably, the Isolated primordial follicle + Alginate treatment was the only one that significantly promoted follicle activation and increased both follicle and oocyte diameters during IVFC, pointing out a higher cell proliferation. In conclusion, the presence of ovarian tissue with or without alginate limited follicle development (activation) after culture. Nevertheless, when primordial follicles were isolated and encapsulated in alginate they presented suitable survival rates, higher rates of follicle activation and continued to grow throughout the culture period.
\end{abstract}

KEYWORDS

3D culture system, alginate, goat, ovarian tissue, primordial follicles

\section{1 | INTRODUCTION}

Preantral follicles (primordial and primary) are the largest ovarian follicle population and represent an important source of potentially competent oocytes for further use in assisted reproductive technologies. Nevertheless, primordial follicles (PFs) are quiescent within the ovary, and usually activate and enter growth phase after exposure to activating or removal of inhibitory stimuli (John, Gallardo, Shirley, \& Castrillon, 2008). However, the mechanisms underlying PF activation and growth are still not well defined.
The in vitro follicle culture (IVFC) is an outstanding tool to study the control of early folliculogenesis. Evidence has suggested that PFs need a stiff, similar than of the ovarian cortex, to initiate their development in vitro (Woodruff \& Shea, 2011). Telfer, McLaughlin, Ding, and Thong (2008) reported that the in vitro culture (IVC) of the ovarian cortical fragments has shown limited results regarding follicle growth. Moreover, differences among fragments in terms of follicular population, number and density of the tissue may occur (Hornick, Duncan, Shea, \& Woodruff, 2012). To minimize the effects of heterogeneity of the ovarian fragments several groups proposed 
to isolate, encapsulate and culture PFs within an inert three-dimensional (3D) biomimetic matrix, such as alginate (Laronda et al., 2014; Shikanov, Smith, Xu, Woodruff, \& Shea, 2011). Despite promising results with secondary and multiple primary follicle culture, this culture system has not demonstrated a proper development of PFs in vitro. Furthermore, there are no experimental data that compares under the same conditions isolated PFs encapsulated in alginate with PFs enclosed in ovarian tissue embedded or not in alginate. Therefore, the present study aims to compare the survival and development rates among isolated PFs embedded in alginate with those enclosed in ovarian fragments in the presence or absence of alginate.

\section{2 | MATERIALS AND METHODS}

\section{1 | Research ethics}

Alternatives to animal testing are the development and implementation of test methods that avoid the use of live animals. One of the major alternatives to in vivo animal testing is in vitro cell culture. In line with this ethical issue, the present study aimed to evaluate the effects of the tested substance (alginate) on in vitro folliculogenesis using caprine follicles recovered from slaughterhouse ovaries. This source of ovarian material represents a by-product of the food industry and is more readily acceptable than euthanasia of animals specifically for scientific purposes.

\subsection{Culture medium and chemicals}

Unless mentioned otherwise, the culture media and other chemicals used in the study were purchased from Sigma Chemical Co. The basic culture medium consisted of $\alpha$-MEM ( $\mathrm{pH} 7.2-7.4)$ supplemented with $1.25 \mathrm{mg} / \mathrm{ml}$ bovine serum albumin, $1 \%$ ITS $(10 \mu \mathrm{g} / \mathrm{ml}$ insulin, $5.5 \mu \mathrm{g} /$ $\mathrm{ml}$ transferrin and $5 \mathrm{ng} / \mathrm{ml}$ selenium), $2 \mathrm{mM}$ glutamine and $2 \mathrm{mM}$ hypoxanthine, which was referred to as $\alpha-\mathrm{MEM}^{+}$.

\section{3 | Experimental protocol}

Ovaries $(n=10)$ were collected at a local slaughterhouse from five adult mixed-breed goats, washed and transported within $1 \mathrm{hr}$ to the laboratory in MEM-HEPES at $4^{\circ} \mathrm{C}$. Ovarian cortex tissue samples from each ovarian pair were cut into 18 slices (approximate size $3 \times 3 \times 1 \mathrm{~mm}$ ) (Figure 1). Twelve of the eighteen fragments were submitted to the mechanical isolation of PFs (Lucci et al., 1999). A total of thirty PFs were isolated per repetition. Groups of 10 follicles were embedded in $10 \mu \mathrm{l}$ drop of alginate (Isolated primordial follicle + Alginate treatment). The remaining six fragments were distributed randomly among the following treatments: Fragment + Alginate treatment (one fragment $/ 20 \mu \mathrm{l}$ drop of alginate) and Fragment alone treatment (fragment without any matrix). The ovarian fragments and isolated follicles were either immediately fixed (Non-cultured control) or in vitro cultured for 1 or 7 days and thereafter fixed for histological analysis as described previously (Lima et al., 2016). Both

\section{Ovarian pair}

(18 Fragments)

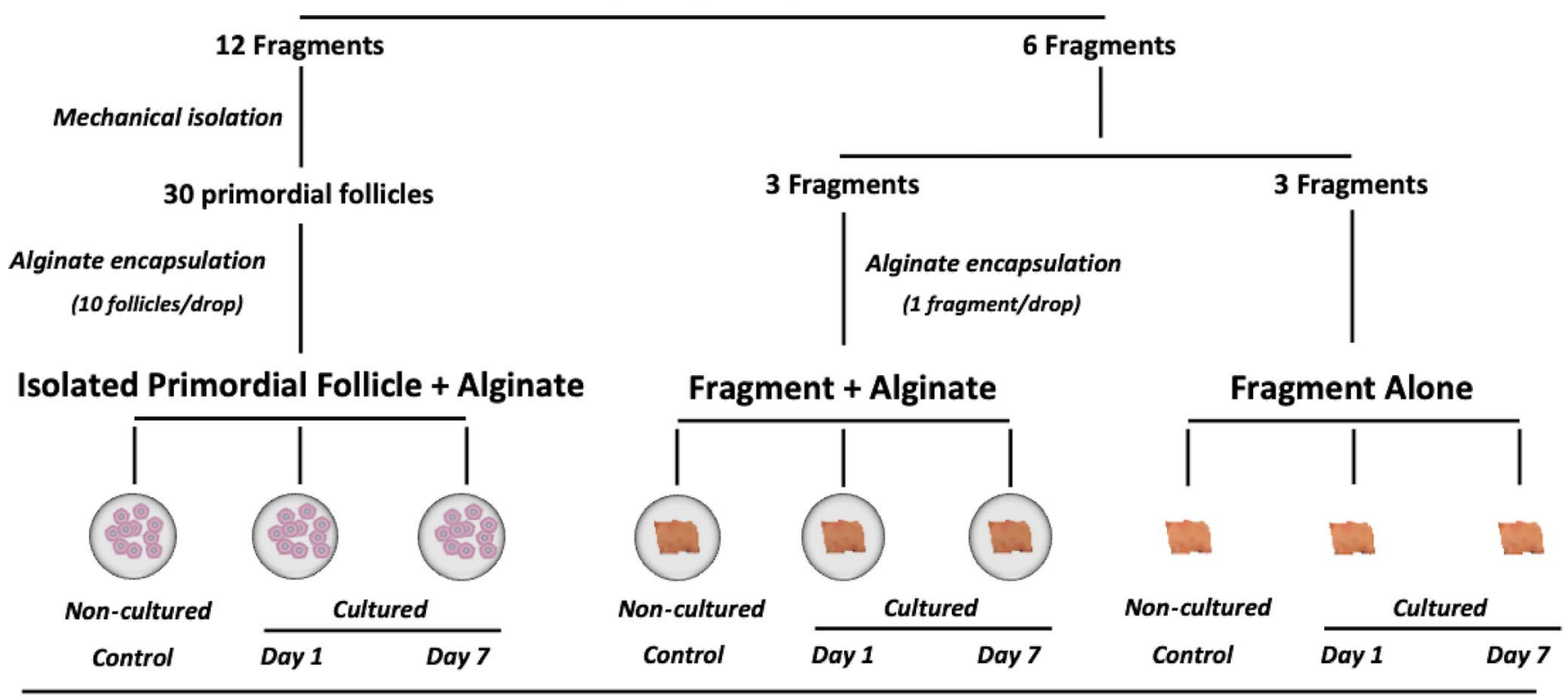

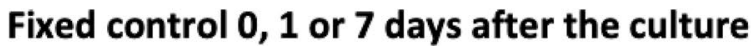

FIGURE 1 Experimental design to assess the effect alginate encapsulation on the development of isolated primordial follicle or enclosed in ovarian tissue after 0,1 or 7 days of IVC 
isolated follicles and the fragments were cultured in 48-well culture dishes containing $500 \mu \mathrm{L}$ of $\alpha-\mathrm{MEM}^{+}$at $39^{\circ} \mathrm{C}$ in $5 \% \mathrm{CO}_{2}$ in air. The experiment was replicated five times, and the culture media were replaced every other day.

\subsection{Follicle and tissue encapsulation in alginate}

For encapsulation, each group of 10 isolated follicles or fragments of ovarian cortex were washed with $100 \mu \mathrm{l}$ drops of alginate to remove the holding medium. Sodium alginate (55\%-65\% guluronic acid) hydrogel was prepared as described in previous reports (Xu, Kreeger, Shea, \& Woodruff, 2006).

\section{5 | Morphological analysis and evaluation of follicular growth in vitro}

Follicle stage and survival were assessed on serial sections. The unilaminar follicles were classified according to their developmental stage as primordial (one layer of flattened granulosa cells or one layer of a mixture of flattened and cuboidal granulosa cells) or primary (from one to less than two complete layers of cuboidal granulosa cells). Also, these follicles were still classified as histologically normal or atretic. To evaluate follicular activation, the percentages of healthy primordial and growing follicles were calculated before and after culture as previously described (Lundy, Smith, O'Connell, Hudson, \& McNatty, 1999). Overall, 150 follicles were evaluated for each group (30 follicles/group/ repetition)

(a)
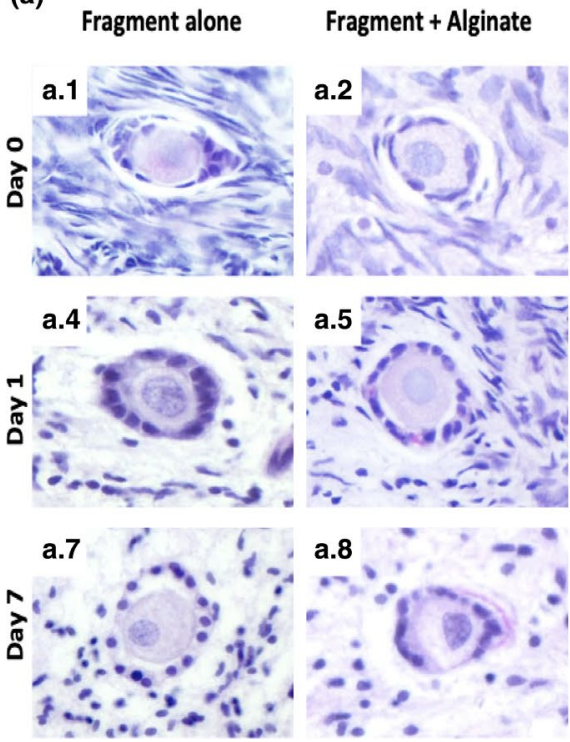

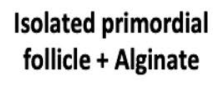

a.3

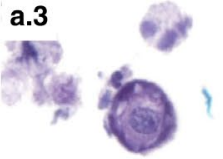

a.6
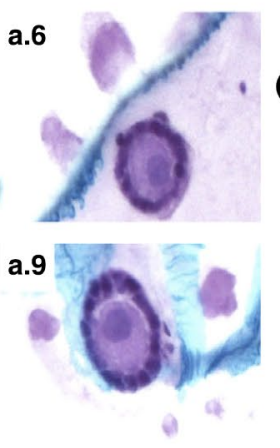

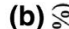

2.6 | Statistical analysis

All statistical analyses were carried out using Sigma Plot 11.0 (Systat Software Inc.). Comparison of means was analysed by ANOVA followed by the Student-Newman-Keuls as a post hoc test, whereas follicular integrity and development were analysed by chi-square test. Data were presented as percentage and mean \pm standard error of mean (SEM), and $p$-value $<.05$ was considered statistically significant.

\section{RESULTS}

Morphological features of normal follicles (MNF) before and after IVFC are shown (Figure 2a). From D1 onwards, the Fragment + Alginate treatment showed a significantly lower percentage of MNF when compared to the other treatments (Figure 2b).

After culture, there was a reduction in the percentage of PFs with a concomitant increase $(p<.05)$ in the percentage of developing follicles (primary follicles) in all treated groups compared to their correspondent controls (Figure 2c). On D7 of culture, the highest $(p<.05)$ percentage of primary follicles was achieved in the isolated primordial culture treatment.

A significant increase in follicle and oocyte diameter was observed in Isolated primordial follicles + Alginate treatment from D0 to D7. In contrast, oocyte diameter decreased from D0 to D7 in the Fragment + Alginate and Fragment alone treatments $(p<.05)$. Only in the Isolated primordial follicles + Alginate treatment the volume filled by granulosa cells in preantral follicles increased from D0 to D7 and was higher than Fragment + Alginate treatment on D7 $(p<.05$; Table 1).

FIGURE 2 Representative images (a), percentage of morphologically normal follicles (b) primordial and primary follicles (c) after 0, 1 or 7 days of IVC. ${ }^{A B}$ Differences among groups within the same day; ${ }^{a b}$ Differences between days within the same group; *Differences from D0 within the same group $(p<.05)$ 


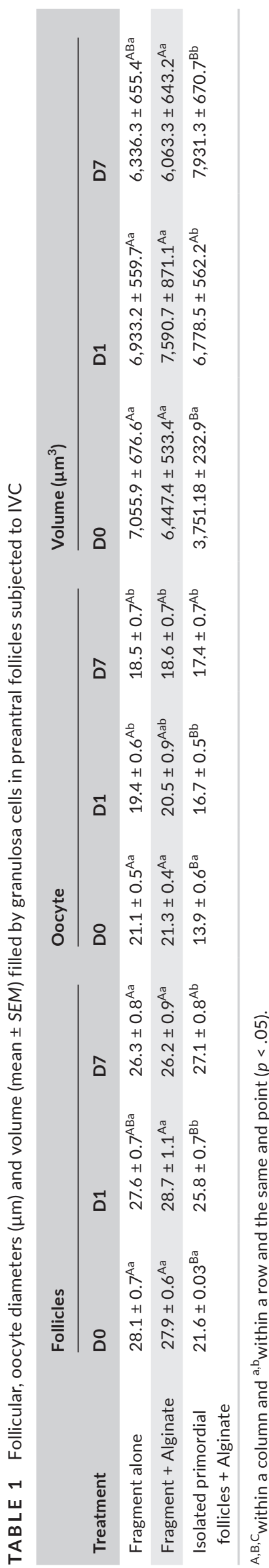

\section{4 | DISCUSSION}

The present study compared for the first time the effect of three culture systems (isolated PFs embedded in alginate matrix with those enclosed in ovarian fragments in the presence or absence of alginate) on caprine PF development in vitro. Our results showed that the use of alginate as a 3D matrix for isolated follicles promoted survival and follicle activation compared to the other two culture systems.

The IVC of follicles in the isolated form within alginate or those cultured only enclosed in ovarian tissue ensured appropriate preservation of follicular morphology. Nevertheless, the IVC of fragments encapsulated in alginate had detrimental effect on the percentage of MNF suggesting that the extra pressure exerted by the alginate on the tissue negatively affected follicle survival, possibly because alginate hydrogels are non-degradable and have a relatively stable elasticity module (Laronda et al., 2014). An additional layer of hydrogel around already dense tissue could also limit the diffusion of nutrients and oxygen towards the cell during IVC further affecting follicle growth and survival.

Isolated follicles demonstrated activation rates 1.3 times higher compared to other treatments. The method of encapsulation of isolated PFs in alginate promoted follicle activation and increased both follicle and oocyte diameters during IVFC. It has been suggested that alginate mimics the role of the extracellular matrix in vivo (Telfer \& Zelinski, 2013), maintaining follicular 3D structure, as well the contact between the oocyte and the surrounding granulosa cells, which facilitated the exchange of substances that are essential for follicular activation and survival (Sadeghnia et al., 2016). In contrast, presence of ovarian tissue around PFs restrained their activation during IVFC, suggesting increasing density and reduced diffusion during culture.

In conclusion, the presence of ovarian tissue limited follicle development during IVC. Nevertheless, when PFs were isolated and encapsulated in alginate they presented suitable survival rates, higher rates of follicle activation and continued to grow throughout the culture period.

\section{ACKNOWLEDGEMENTS}

Correia's scholarship and this research were supported by grants from the National Council for Scientific and Technological Development (CNPq - N 407594/2013-2).

\section{CONFLICT OF INTEREST}

None of the authors have any conflict of interest to declare.

\section{AUTHOR CONTRIBUTIONS}

In this study, the co-authors: H. H. V. Correia, L. F. Lima, F. G. C. Sousa, A. C. A. Ferreira, J. Cadenas and V. M. Paes performed the experimental protocols, acquisition of data and participated in drafting the full manuscript. Bênner Geraldo Alves contributed to the analysis of the data. A. Shikanov and J. R. Figueiredo participated in substantial contribution to conception and revising it critically for important intellectual content. All the authors in this manuscript have read and approved the final version. 


\section{ORCID}

José Ricardo Figueiredo (iD https://orcid.

org/0000-0002-5139-0964

\section{DATA AVAILABILITY}

The data that support the findings of this study are available from the corresponding author upon reasonable request.

\section{REFERENCES}

Hornick, J. E., Duncan, F. E., Shea, L. D., \& Woodruff, T. K. (2012). Isolated primate primordial follicles require a rigid physical environment to survive and grow in vitro. Human Reproduction, 27, 1801-1810. https ://doi.org/10.1093/humrep/der468

John, G. B., Gallardo, T. D., Shirley, L. J., \& Castrillon, D. H. (2008). Foxo3 is a PI3K-dependent molecular switch controlling the initiation of oocyte growth. Developmental Biology, 1, 197-204. https://doi. org/10.1016/j.ydbio.2008.06.017

Laronda, M. M., Duncan, F. E., Hornick, J. E., Xu, M., Pahnke, J. E., Whelan, K. A., ... Woodruff, T. K. (2014). Alginate encapsulation supports the growth and differentiation of human primordial follicles within ovarian cortical tissue. Journal of Assisted Reproduction and Genetics, 31, 1013-1028. https://doi.org/10.1007/s10815-014-0252-x

Lima, L. F., Rochaa, R. M. P., Alves, A. M. C. V., Carvalho, A. A., Chaves, R. N., Lopes, C. A. P., ... Figueiredo, J. R. (2016). Comparison between the additive effects of diluted ( $\mathrm{rFSH}$ ) and diluted/dynamized (FSH 6 $\mathrm{CH}$ ) recombinant follicle-stimulating hormone on the in vitro culture of ovine preantral follicles enclosed in ovarian tissue. Complement Ther Med, 25, 39-44.

Lucci, C. M., Amorim, C. A., Báo, S. N., Figueiredo, J. R., Rodrigues, A. P., ...Gonçalves, P. B. (1999). Effect of the interval of serial sections of ovarian tissue in the tissue chopper on the number of isolated caprine preantral follicles. Animal Reproduction Science, 56, 39-49. https://doi.org/10.1016/S0378-4320(99)00031-7

Lundy, T., Smith, P., O'Connell, A., Hudson, N. L., \& McNatty, K. P. (1999). Populations of granulosa cells in small follicles of the sheep ovary.
Journal of Reproduction and Fertility, 115, 251-262. https://doi. org/10.1530/jrf.0.1150251

Sadeghnia, S., Akhondi, M. M., Hossein, G., Mobini, S., Hosseini, L., Naderi, M. M., \& Shirazi, A. (2016). Development of sheep primordial follicles encapsulated in alginate or in ovarian tissue in fresh and vitrified samples. Cryobiology, 72, 100-105. https://doi.org/10.1016/j. cryobiol.2016.03.001

Shikanov, A., Smith, R. M., Xu, M., Woodruff, T. K., \& Shea, L. D. (2011). Hydrogel network design using multifunctional macromers to coordinate tissue maturation in ovarian follicle culture. Biomaterials, 32, 2524-2531. https://doi.org/10.1016/j.biomaterials.2010.12.027

Telfer, E. E., McLaughlin, M., Ding, C., \& Thong, K. J. (2008). A twostep serum-free culture system supports development of human oocytes from primordial follicles in the presence of activin. Human Reproduction., 23, 1151-1158. https://doi.org/10.1093/humrep/ den070

Telfer, E. E., \& Zelinski, M. B. (2013). Ovarian follicle culture: Advances and challenges for human and nonhuman primates. Fertility and Sterility, 99, 1523-1533. https://doi.org/10.1016/j.fertnstert.2013.03.043

Woodruff, T. K., \& Shea, L. D. (2011). A new hypothesis regarding ovarian follicle development: Ovarian rigidity as a regulator of selection and health. Journal of Assisted Reproduction and Genetics, 28, 3-6. https:// doi.org/10.1007/s10815-010-9478-4

Xu, M., Kreeger, P. K., Shea, L. D., \& Woodruff, T. K. (2006). Tissueengineered follicles produce live, fertile offspring. Tissue Engineering, 12, 2739-2746. https://doi.org/10.1089/ten.2006.12.2739

How to cite this article: Correia HHV, Lima LF, Sousa FGC, et al. Activation of goat primordial follicles in vitro: Influence of alginate and ovarian tissue. Reprod Dom Anim. 2020;55: 105-109. https://doi.org/10.1111/rda.13582 